\title{
Catheter-Based Renal Denervation Attenuates Kidney Interstitial Fibrosis in a Canine Model of High-Fat Diet-Induced Hypertension
}

\author{
Liying Gong $^{\text {a, b }}$ Fenglin Jiang ${ }^{a} \quad$ Zhihui Zhang $^{a} \quad$ Xinguo Wang ${ }^{a} \quad \mathrm{Hui} \mathrm{Li}^{\mathrm{a}}$ \\ Yan Kuang $^{\text {a }}$ Guoping Yang ${ }^{b}$ \\ aDepartment of Cardiology, The Third Xiangya Hospital of Central South University, \\ Changsha, PR China; ${ }^{b}$ Department of Clinical Pharmacology Center, \\ The Third Xiangya Hospital of Central South University, Changsha, PR China
}

\section{Keywords}

Catheter-based renal denervation - Hypertension - Renal sympathetic nerve

Renin-angiotensin-aldosterone system $\cdot$ Kidney interstitial fibrosis

\begin{abstract}
Background/Aims: Catheter-based renal denervation (RDN) has emerged as an innovative interventional approach for reducing blood pressure (BP), suppressing ventricular substrate remodeling, and attenuating heart failure, which suggests that it might reduce kidney fibrosis in a canine model of high-fat diet-induced hypertension. This study thus sought to assess whether RDN could reduce kidney fibrosis and halt the progression of renal impairment in a canine model of high-fat diet-induced hypertension. Methods: Thirty-two beagles were randomized into either the normal control group (normal diet, $n=10$ ) or the hypertension group (high-fat diet, $n=22$ ). After successful establishment of the model, the hypertension model group was randomized to either the RDN group $(n=9)$ or the sham-surgery group $(n=8)$. Renal artery angiography, $\mathrm{BP}$, heart rate $(\mathrm{HR})$, and blood and urine biochemistry results were assessed at 1, 3, and 6 months after surgery. Canines were sacrificed at 6 months after surgery. The extent of kidney interstitial fibrosis, transforming growth factor-beta 1, alpha-smooth muscle actin, connective tissue growth factor, and E-cadherin protein were measured. $\boldsymbol{R e}$ sults: The group fed a high-fat diet had significantly $(p<0.05)$ increased body weight, BP, and $\mathrm{HR}$ and higher levels of urine albumin, serum noradrenaline (NE), and angiotensin II (Angll) than the control group. The sham-surgery group and RDN group also had higher levels than
\end{abstract}




\section{Kidney \\ Blood Pressure \\ Research}

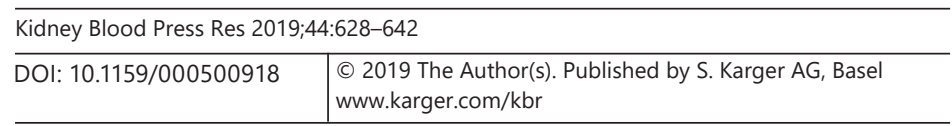

Gong et al.: Cather-Based RDN Attenuates Kidney Interstitial Fibrosis

the control group $(p<0.05)$. Compared with the sham-surgery group, the RDN group had lower BP, urine albumin, serum NE, and Angll and less fibrotic tissue (all $p<0.05$ ). Conclusion: $\mathrm{RDN}$ reduced $\mathrm{BP}$, slowed progression of albuminuria, and suppressed renal remodeling in a canine model of high-fat diet-induced hypertension.

(C) 2019 The Author(s)

Published by S. Karger AG, Basel

\section{Introduction}

Obesity is widely recognized as a risk factor for hypertension, diabetes, and other comorbid conditions that contribute to the development of chronic kidney disease (CKD) [1, 2]. Structural changes in the kidneys occur within a few weeks after consumption of a high-fat diet [3]. Jeffery first found that dogs fed on a high-fat diet for only 7-9 weeks demonstrated significant functional, biochemical, and histologic changes in the kidney [4]. The mechanisms by which obesity caused kidney structural changes are not fully understood; however, they likely involved a combination of metabolic, inflammatory, and hemodynamic changes.

Activation of the sympathetic nervous system (SNS) and the renin-angiotensin-aldosterone system (RAAS) may contribute to hypertension. Hypertension, which along with inflammation, glomerular hyperfiltration, and metabolic derangements, may cause renal injury. Prominent pathological features of patients with kidney disease are inflammation, tubular atrophy, and renal fibrosis [5]. However, fibrosis is the typical characteristic used to determine the prognosis of the disease. Complex molecular interactions and cellular networks regulate the fibrotic process. Angiotensin II (AngII), the main peptide of the renin-angiotensin system, is well known to participate in the pathogenesis of renal diseases through the regulation of inflammation and fibrosis, which are 2 key processes [6]. After AngII activation, renal tubular epithelial cells can regulate and increase the expression of endogenous profibrogenic cytokine transforming growth factor- $\beta 1$ (TGF- $\beta 1$ ), which is a stronger fibrogenic factor [6]. Connective tissue growth factor (CTGF) is a downstream response element of AngII and TGF- $\beta 1$ that is the main mediator of renal fibrosis [7-9]. TGF- $\beta 1$-induced trans-differentiation of renal tubular epithelial cells can also decrease the expression of E-cadherin and increase the expression of epidermal growth factor, resulting in the production of alphasmooth muscle actin ( $\alpha$-SMA) [10].

Catheter-based renal denervation (RDN) is an invasive procedure involving the percutaneous introduction of a catheter into the renal arteries. This procedure targets the sympathetic nerve fibers in the renal arteries, by applying radiofrequency energy to the kidney blood vessel wall to damage the nerve fibers (including afferent and efferent) surrounding the artery [11-13]. Increased sympathetic nerve traffic is detrimental in the setting of hypertension and progression of renal disease [14-16]. RDN has been shown to reduce blood pressure (BP) in obese patients. However, whether RDN can suppress renal remodeling and halt the progression of renal impairment is unknown. Therefore, we sought to evaluate the therapeutic effects of RDN on CKD progression and renal remodeling in a canine model of high-fat diet-induced hypertension.

\section{Materials and Methods}

Animals Preparation

Thirty-two beagles of the same species (aged 10-12 months; 16 of each sex) were purchased from the Shanghai Experimental Animal Center (Shanghai, China). They were housed in a single cage at the Xiangya Animal Center of Central South University (Changsa, China). After 1 week of adaptive feeding, they were 


\section{Kidney \\ Blood Pressure Research}

\begin{tabular}{l|l}
\hline Kidney Blood Press Res 2019:44:628-642 \\
\hline DOI: 10.1159/000500918 & $\begin{array}{l}\text { @ 2019 The Author(s). Published by S. Karger AG, Basel } \\
\text { www.karger.com/kbr }\end{array}$ \\
\hline
\end{tabular}

Gong et al.: Cather-Based RDN Attenuates Kidney Interstitial Fibrosis

randomly divided into the normal control group (normal diet, $n=10$ ) or the hypertension model group (fed a high-fat diet, $n=22$ ). They all underwent bilateral renal artery angiography so the renal artery anatomy could be examined. According to the protocol, all canines were fed adult dog food and vitamin/mineral supplements. Canines in the control group were fed on a regular diet, and those canines in the hypertension model group were fed on a high-fat diet (0.3-0.4 kg of lard was added to the regular diet of $250 \mathrm{~g}$ every day). After 12 weeks of the high-fat diet, 20 beagles had an approximately $50 \%$ increase in body weight. After successful establishment of the model and the application of radiofrequency for RDN, animals in the RDN and sham-surgery groups were fed a diet containing $0.1 \mathrm{~kg}$ of fat every day to maintain their body weight after the BP of the canines became stable. The hypertension model group was then divided into the RDN group and sham-surgery group.

Five beagles were excluded from the study for the following reasons: 2 canines in the hypertension model group did not satisfy the criteria of hypertension (increases in BP $<15 \mathrm{~mm} \mathrm{Hg}$ ); 2 canines died due to anesthesia complications; and one canine developed a retroperitoneal hematoma caused by femoral artery puncture. The RDN group $(n=9)$ underwent RDN; the sham-surgery group $(n=8)$ and control group $(n=10)$ underwent renal angiography. Renal artery angiography was performed again at 3 and 6 months after surgery. The beagles were sacrificed under deep anesthesia 6 months after surgery. This study was conducted in compliance with the guidelines of the National Institutes of Health for the care and use of laboratory animals and approved by the animal studies subcommittee of the Third Xiangya Hospital of Central South University.

\section{RDN and Renal Artery Angiography}

We have previously reported the details of the RDN and renal artery angiography performed at our center [17]. Briefly, after an overnight fast, the skin areas of the back and bilateral femoral artery areas of the canines were prepared, followed by an intramuscular injection of Zoletil ${ }^{\circledR}(7 \mathrm{mg} / \mathrm{kg}$; Virbac, Carros, France) and Sumianxin $(0.1 \mathrm{mg} / \mathrm{kg}$; Jilin Shengda Animal Drug Co., Ltd., Yanbian, Jilin, China) for anesthesia. After successful anesthetization, the canines were immobilized on the surgery table in the supine position. An ablation electrode was placed on the back and connected to a radiofrequency ablation device (IBI; St. Jude Medical, Inc., St. Paul, MN, USA). The temperature was controlled at approximately $55^{\circ} \mathrm{C}$, and the energy was set at $80 \mathrm{~W}$. The right femoral artery area was conventionally disinfected, and guided renal artery angiography was performed to detect renal artery stenosis. Renal artery angiography was performed again at 1, 3, and 6 months.

Measurements of Body Weight, $B P$, and Heart Rate

The body weight of each canine (with an empty stomach) was determined in the morning by using the electronic weighting scales. Systolic BP, diastolic BP, mean BP, and heart rate (HR) were measured using the tail-cuff method using a noninvasive animal BP instrument (BP-10E; Beijing Softrong Biotechnology Co., Beijing, China). Plasma concentration and norepinephrine (NE) levels in renal tissues were measured by using high-performance liquid chromatography with electrochemical detection (HTEC-500; Eicom Corporation, Tokyo, Japan).

Blood and Tissue Biochemistry Test

After an overnight fast, the canines were placed in the holder. Blood sample was collected as previously described [17]: they were collected from the small saphenous vein in the lateral hind legs at baseline, before surgery, and 1, 3, and 6 months after surgery. Blood and tissue AngII levels were measured using a radioimmunoassay kit ( ${ }^{125}$ AngiotensinII radioimmunoassay assay; Chemclin Biotech Co., Ltd., Beijing, China) according to the manufacturer's manual. Blood and tissue NE levels were determined and quantified by highperformance liquid chromatography, as described previously [18].

Urine Biochemistry Test

At baseline, before surgery, and 1, 3, and 6 months after surgery, urine samples were collected by vesicopuncture and used to determine the urine albumin levels.

Western Blot and Immunohistochemistry Detection of TGF- $\beta 1, \alpha-S M A, C T G F$, and E-Cadherin Expression

TGF- $\beta 1, \alpha$-SMA, E-cadherin, and CTGF protein expressions in kidney tissue were measured by Western blot at 6 months after surgery. The membranes were probed with rabbit antibodies against TGF- $\beta 1$ (1:500 dilution; Cat. No. sc-146; Santa Cruz, Biotechnology, Dallas, TX, USA), $\alpha$-SMA (1:300 dilution; Cat. No. ab5697; Abcam, Cambridge, UK), E-cadherin protein (1:400 dilution; Cat. No. orb11101; biorbyt, San Francisco, CA, 
Table 1. Weight, BP, and HRs of the 3 groups pre- and post-surgery

\begin{tabular}{|c|c|c|c|}
\hline Groups & $\begin{array}{l}\text { Control group } \\
(n=10)\end{array}$ & $\begin{array}{l}\text { RDN group } \\
(n=9)\end{array}$ & $\begin{array}{l}\text { Sham-surgery group } \\
(n=8)\end{array}$ \\
\hline \multicolumn{4}{|l|}{ Body weight, kg } \\
\hline Baseline & $12.28 \pm 1.89$ & $11.87 \pm 0.87$ & $12.38 \pm 0.54$ \\
\hline Presurgery & $13.14 \pm 1.24$ & $17.60 \pm 2.00 *$, \# & $17.55 \pm 2.71^{*, \#}$ \\
\hline 6-month post-surgery & $13.73 \pm 1.85$ & $18.66 \pm 2.40^{*}, \#$ & $18.61 \pm 2.03^{*, \#}$ \\
\hline \multicolumn{4}{|l|}{$\mathrm{BP}, \mathrm{mm} \mathrm{Hg}$} \\
\hline \multicolumn{4}{|l|}{$\mathrm{SBP}, \mathrm{mm} \mathrm{Hg}$} \\
\hline Baseline & $125.70 \pm 6.00$ & $123.67 \pm 9.96$ & $124.88 \pm 8.22$ \\
\hline Presurgery & $129.80 \pm 6.81$ & $153.22 \pm 9.48^{*, \#}$ & $151.50 \pm 12.27^{*}, \#$ \\
\hline 1-month post-surgery & $130.80 \pm 6.60$ & $134.44 \pm 8.52^{\psi}$ & $146.75 \pm 13.72^{\#}$ \\
\hline 3-month post-surgery & $132.40 \pm 13.34$ & $135.33 \pm 10.27 \Psi$, ๆ & $152.50 \pm 12.69 *, \#$ \\
\hline 6-month post-surgery & $127.80 \pm 3.68$ & $129.67 \pm 8.51^{\psi, \eta}$ & $156.00 \pm 8.60 *, \#$ \\
\hline \multicolumn{4}{|l|}{$\mathrm{DBP}, \mathrm{mm} \mathrm{Hg}$} \\
\hline Baseline & $73.70 \pm 5.98$ & $77.11 \pm 9.58$ & $76.00 \pm 8.59$ \\
\hline Presurgery & $75.30 \pm 10.61$ & $92.56 \pm 7.63^{*, \#}$ & $95.13 \pm 9.09 *, \#$ \\
\hline 1-month post-surgery & $77.60 \pm 13.37$ & $82.89 \pm 6.92^{\psi}$ & $93.13 \pm 12.16^{*, \#}$ \\
\hline 3-month post-surgery & $81.60 \pm 4.81$ & $82.45 \pm 4.36^{\psi, ~ ๆ ~}$ & $91.63 \pm 9.74 *, \#$ \\
\hline 6-month post-surgery & $78.20 \pm 7.21$ & $79.33 \pm 5.32^{\psi, ~ ๆ ~}$ & $94.38 \pm 18.43^{*, \#}$ \\
\hline \multicolumn{4}{|l|}{$\mathrm{MBP}, \mathrm{mm} \mathrm{Hg}$} \\
\hline Baseline & $93.80 \pm 6.09$ & $92.56 \pm 9.28$ & $92.38 \pm 7.91$ \\
\hline Presurgery & $94.80 \pm 7.52$ & $112.78 \pm 7.76^{*, \#}$ & $114.25 \pm 9.53^{*, \#}$ \\
\hline 1-month post-surgery & $95.40 \pm 10.25$ & $100.22 \pm 6.44^{\psi}$ & $110.88 \pm 12.19 *, \#$ \\
\hline 3-month post-surgery & $98.40 \pm 5.66$ & $100.78 \pm 5.61^{\psi, 9}$ & $108.75 \pm 10.05^{*}, \#$ \\
\hline 6-month post-surgery & $98.20 \pm 6.09$ & $96.78 \pm 6.85^{\psi, \text { ๆ }}$ & $114.88 \pm 14.53^{*, \#}$ \\
\hline \multicolumn{4}{|l|}{$\mathrm{HR}, \mathrm{bpm}$} \\
\hline Baseline & $132.30 \pm 19.48$ & $131.56 \pm 10.92$ & $129.63 \pm 11.65$ \\
\hline Presurgery & $124.20 \pm 6.07$ & $143.67 \pm 14.30 *$ & $141.50 \pm 13.58^{*}$ \\
\hline 1-month post-surgery & $122.90 \pm 17.53$ & $136.22 \pm 12.79$ & $136.63 \pm 8.67$ \\
\hline 3-month post-surgery & $125.40 \pm 18.25$ & $132.44 \pm 15.04$ & $140.38 \pm 17.24$ \\
\hline 6-month post-surgery & $124.00 \pm 21.49$ & $131.11 \pm 22.17$ & $144.13 \pm 18.14$ \\
\hline
\end{tabular}

$* p<0.05$, vs. the control group at the same time point.

${ }^{\#} p<0.05$, vs. baseline of the same group.

${ }^{\Psi} p<0.05$, vs. presurgery of the same group.

I $p<0.05$, vs. the sham-surgery group at the same time point.

$\mathrm{HR}$, heart rate; $\mathrm{BP}$, blood pressure; bpm, beats per minute; DBP, diastolic blood pressure; MBP, mean blood pressure; SBP, systolic blood pressure.

USA), and CTGF (1:800 dilution; Cat. No. ab125943; Abcam). All blots were developed using enhanced chemiluminescence (GE Healthcare Bio-Sciences Corp., Piscataway, NJ, USA). Autoradiographic films were scanned using densitometry and quantified using Image Quant 3.3 software.

Expressions of TGF- $\beta 1, \alpha$-SMA, and E-cadherin protein in the kidney tissue were detected using rabbit antibodies against TGF- $\beta 1$ (1:200 dilution; Cat. No. sc-146; Santa Cruz Biotechnology), $\alpha$-SMA (1:200 dilution; Cat. No. ab5697; Abcam), and E-cadherin protein (1:300 dilution; Cat. No. orb11101; biorbyt). Formalin-fixed paraffin-embedded tissue sections were deparaffinized with xylene. Deparaffinized sections were incubated with the primary antibody overnight at $4{ }^{\circ} \mathrm{C}$ and then with a biotinated goat anti-rabbit IgG antibody. Immunoreactivity was visualized with an avidin-biotin-peroxidase kit from Vector Laboratories.

For quantification of TGF- $\beta 1, \alpha$-SMA and E-cadherin, images morphometry of TGF- $\beta 1, \alpha$-SMA and E-cadherin were analyzed using Image pro-Plus6.0 (Media Cybernetics, Inc., Rockville, MD, USA) under 200× magnification. Three sections were chosen from each canine and 10 consecutive fields of renal cortex were randomly chosen from each section. Positive stained areas (brown or yellow) were measured in each field. The mean value was used for the analysis. 
Table 2. Plasma NE and AngII levels in different groups at different time points

\begin{tabular}{|c|c|c|c|c|c|c|}
\hline \multirow[t]{2}{*}{ Groups } & \multicolumn{3}{|c|}{ Plasma NE levels, pg/mL } & \multicolumn{3}{|c|}{ Plasma AngII levels, pg/mL } \\
\hline & baseline & presurgery & $\begin{array}{l}\text { 6-month } \\
\text { post-surgery }\end{array}$ & baseline & presurgery & $\begin{array}{l}\text { 6-month } \\
\text { post-surgery }\end{array}$ \\
\hline Control group $(n=10)$ & $123.36 \pm 80.15$ & $141.13 \pm 52.16$ & $180.81 \pm 77.03$ & $66.48 \pm 5.19$ & $67.84 \pm 8.03$ & $69.33 \pm 2.58$ \\
\hline RDN group $(n=9)$ & $106.12 \pm 52.00$ & $531.07 \pm 159.58^{*,} \#$ & $299.45 \pm 97.08 \psi$ & $63.31 \pm 7.19$ & $122.74 \pm 10.78^{*}, \#$ & $88.49 \pm 5.69^{\#,} \Psi$, ๆ \\
\hline Sham-surgery group $(n=8)$ & $140.16 \pm 70.63$ & $553.29 \pm 202.98^{*}, \#$ & $609.19 \pm 144.36^{*, \#}$ & $63.40 \pm 8.80$ & $130.40 \pm 8.30 *, \#$ & $136.20 \pm 6.27^{*, \#}$ \\
\hline
\end{tabular}

$* p<0.05$ vs. the control group at the same time point.

$\# p<0.05$ vs. baseline of the same group.

$\psi_{p<0.05 \text { vs. presurgery of the same group. }}^{p}$

ๆ $p<0.05$ vs. the sham-surgery group at the same time point.

AngII, angiotensin II; NE, norepinephrine; RDN, renal denervation.

Histological and Electron Microscopic Analysis

Left and right sections of the renal cortex were dissected from the kidney and immediately stored at $-80{ }^{\circ} \mathrm{C}$. Hematoxylin and eosin staining and Masson's trichrome staining and electron microscopic examination of the kidney tissue (electron microscopy laboratory of the Pathology Department of Xiangya Hospital, Central South University) were performed to identify increased concentrations of interstitial fibrosis. For quantification of collagen, image morphometry of Masson's trichrome was measured by using Image pro-Plus6.0 (Media Cybernetics, Inc., Rockville, MD, USA) under 200× magnification. Three sections were chosen from each canine and 10 consecutive fields of renal cortex (avoiding large vessels and glomeruli) were randomly chosen from each section. Blue-stained interstitial fibrotic areas were measured in each field. The mean value was used for the analysis.

\section{Statistical Analyses}

Data were analyzed using SPSS 21.0 (SPSS; IBM, Armonk, NY, USA). Continuous variables are presented as mean \pm SD. A 2 -sample independent Student $t$ test was used to compare means between 2 groups, and an analysis of variance involving the Newman-Keuls tests was used to compare the means of continuous variables among multiple groups. In the case of a significant difference, a further analysis was undertaken with a Tukey-Kramer test. In all cases, $p<0.05$ was considered to be significant. All figures were created with GraphPad Prism version 5 (GraphPad, San Diego, CA, USA).

\section{Results}

\section{Analyses of the General Parameters}

As shown in Table 1, there were no significant differences at baseline in body weight, BP, and HR among the 3 groups at baseline. However, after 3 months of a high-fat diet, body weight and BP were significantly higher than the baseline values in the hypertension group and the control groups $(p<0.05)$. However, after 3 months of the high-fat diet, no significant differences were found for these parameters between the sham-surgery group and the RDN group. The parameters of systolic BP, diastolic BP, and mean BP were significantly decreased at 1,3 , and 6 months compared to the values before surgery (all $p<0.05$ ).

\section{Plasma Concentrations and Renal Tissue Levels of NE and AngII}

As shown in Table 2, compared with the baseline values, plasma levels of NE and AngII were markedly increased in the RDN and sham-surgery groups 3 months after being fed a high-fat diet (all $p<0.05$ ). The plasma NE and AngII levels of the RDN group were significantly decreased compared to the levels of the sham-surgery group (all $p<0.05$ ) and the presurgery plasma NE and AngII levels (all $p<0.05$ ) 6 months after surgery. As shown in 
Table 3. Kidney tissue NE and AngII levels in different groups at 6-month post-surgery

\begin{tabular}{lll}
\hline Groups & $\begin{array}{l}\text { Kidney tissue NE levels, } \\
\mu \mathrm{g} / \mathrm{g}\end{array}$ & $\begin{array}{l}\text { Kidney tissue AngII levels, } \\
\mathrm{pg} / \mathrm{mg}\end{array}$ \\
\hline Control group $(n=10)$ & $1,643.31 \pm 374.60$ & $1.48 \pm 0.05$ \\
RDN group $(n=9)$ & $1,888.38 \pm 284.16^{\#}$ & $1.78 \pm 0.43^{\#}$ \\
Sham-surgery group $(n=8)$ & $2,284.26 \pm 268.75^{*}$ & $2.27 \pm 0.12^{*}$ \\
\hline${ }^{*} p<0.05$ vs the control group. & \\
${ }^{\#} p<0.05$ vs. the sham-surgery group. & \\
AngII, angiotensin II; NE, norepinephrine; RDN, renal denervation. & \\
\hline
\end{tabular}

Table 4. Urine albumin levels in different groups at different time points

\begin{tabular}{|c|c|c|c|c|}
\hline \multirow[t]{2}{*}{ Groups } & \multicolumn{4}{|c|}{ Urine albumin } \\
\hline & baseline & presurgery & $\begin{array}{l}\text { 3-month post- } \\
\text { surgery }\end{array}$ & $\begin{array}{l}\text { 6-month post- } \\
\text { surgery }\end{array}$ \\
\hline Control group $(n=10)$ & $5.64 \pm 2.46$ & $6.14 \pm 1.95$ & $5.94 \pm 2.31$ & $5.88 \pm 2.27$ \\
\hline RDN group $(n=9)$ & $5.79 \pm 2.07$ & $14.28 \pm 4.71^{*, \#}$ & $15.08 \pm 1.96^{*, \#, \text { ๆ }}$ & $16.24 \pm 1.96^{*, \#, \text { ๆ }}$ \\
\hline Sham-surgery group $(n=8)$ & $5.14 \pm 2.13$ & $12.05 \pm 2.29 *$, \# & $20.88 \pm 3.96^{*, \#, \Psi}$ & $25.40 \pm 1.64^{*}, \#, \Psi, \S$ \\
\hline $\begin{array}{l}{ }^{*} p<0.05 \text { vs. the control } \\
{ }^{\#} p<0.05 \text { vs. baseline of } t \\
{ }^{\Psi} p<0.05 \text { vs. presurgery } \\
{ }^{\top} p<0.05 \text { vs. the sham-su } \\
{ }^{\S} p<0.05 \text { vs. } 3 \text {-month pos } \\
\text { RDN, renal denervation. }\end{array}$ & $\begin{array}{l}\text { up at the sam } \\
\text { same group. } \\
\text { he same grou } \\
\text { ry group at } t \\
\text { urgery of the }\end{array}$ & $\begin{array}{l}\text { p. } \\
\text { le same time poi } \\
\text { same group. }\end{array}$ & & \\
\hline
\end{tabular}

Table 3, at 6 months after surgery, the renal tissue NE and AngII levels of the sham-group were significantly increased compared to the levels of the control group (all $p<0.05$ ); however, the NE and AngII levels of the RDN group were significantly decreased when compared with the levels of the sham-surgery group (all $p<0.05$ ).

\section{Urine Albumin Levels before and after Surgery}

As shown in Table 4, compared with the baseline values, the urine albumin levels were markedly increased 3 months after the high-fat diet in the RDN and sham-surgery groups (all $p<0.05$ ). Compared to the levels of the sham-surgery group, the urine albumin levels of the RDN group were significantly decreased at 3 and 6 months after surgery (all $p<$ $0.05)$.

\section{Expressions of TGF- $\beta 1, \alpha$-SMA, E-Cadherin, and CTGF Protein in the Kidney Tissue 6}

Months after Surgery

Expression of TGF- $\beta 1$ Protein

Western blotting test results showed that at 6 months post-surgery, differences in the expression of TGF- $\beta 1$ protein existed among the 3 groups (Fig. $1 \mathrm{~A}, \mathrm{~B}$; online suppl. Table 1; for all online suppl. material, see www.karger.com/doi/10.1159/000500918). The RDN and the sham-surgery groups showed a higher expression of TGF- $\beta 1$ than that in the control group; however, the sham-surgery group exhibited the highest expression. 


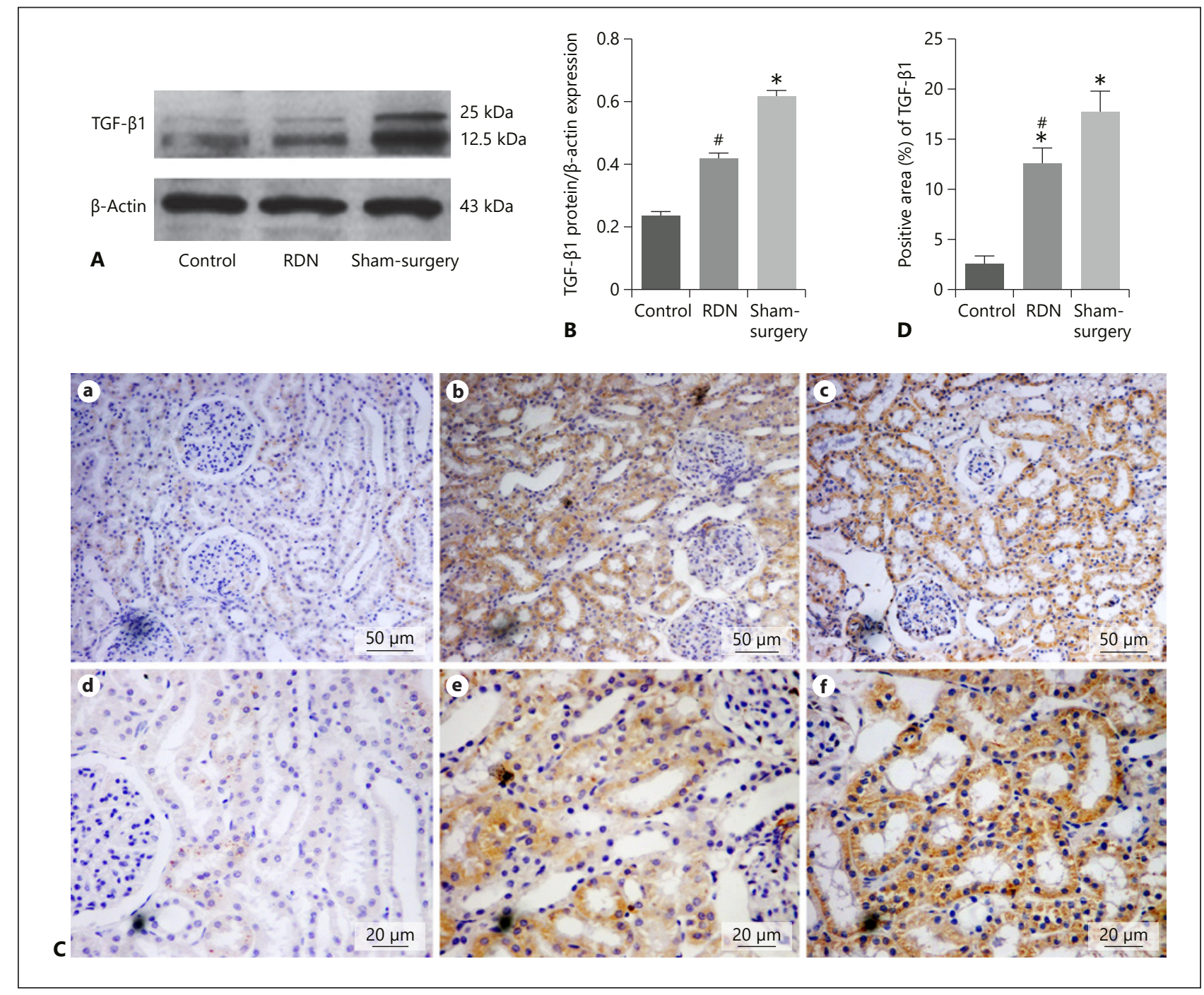

Fig. 1. Effect of RDN and high-fat diet induced hypertension on the expression of renal TGF- $\beta 1$. A, B TGF- $\beta 1$ protein expression in canine kidney tissue in different groups is assessed by western blotting. $\mathbf{C}$ Immunohistochemistry (a-c 200x; d-f 400x) and (D) quantification results of TGF- $\beta 1$ in canine kidney tissue in Controls, RDN, and Sham-surgery groups 6 months post-surgery. $n=5$, data are presented as mean \pm SD. ${ }^{*} p<$ 0.05 , vs. control group. ${ }^{\#} p<0.05$ vs. sham-surgery group. TGF- $\beta 1$, transforming growth factor- $\beta 1$; RDN, renal denervation.

Immunohistochemical results (Fig. 1C) indicated that the immunoreactive products of TGF- $\beta 1$ (brown and yellow) were mainly localized in the cytoplasm and membrane of renal tubular epithelial cells. Both the RDN and sham-surgery groups had exhibited positive expression, and the sham-surgery group exhibited the strongest expression, and the positive surface density was higher than in the RDN group (Fig. 1D).

\section{Expression of $\alpha$-SMA Protein}

As shown in Figure 2A, B and online supplementary Table 1, western blot test results showed that 6 months post-surgery, differences in the expression of $\alpha$-SMA protein existed among the 3 groups. The RDN and the sham-surgery groups showed a higher expression of $\alpha$-SMA than that in the control group; however, the sham-surgery group exhibited the highest expression. 


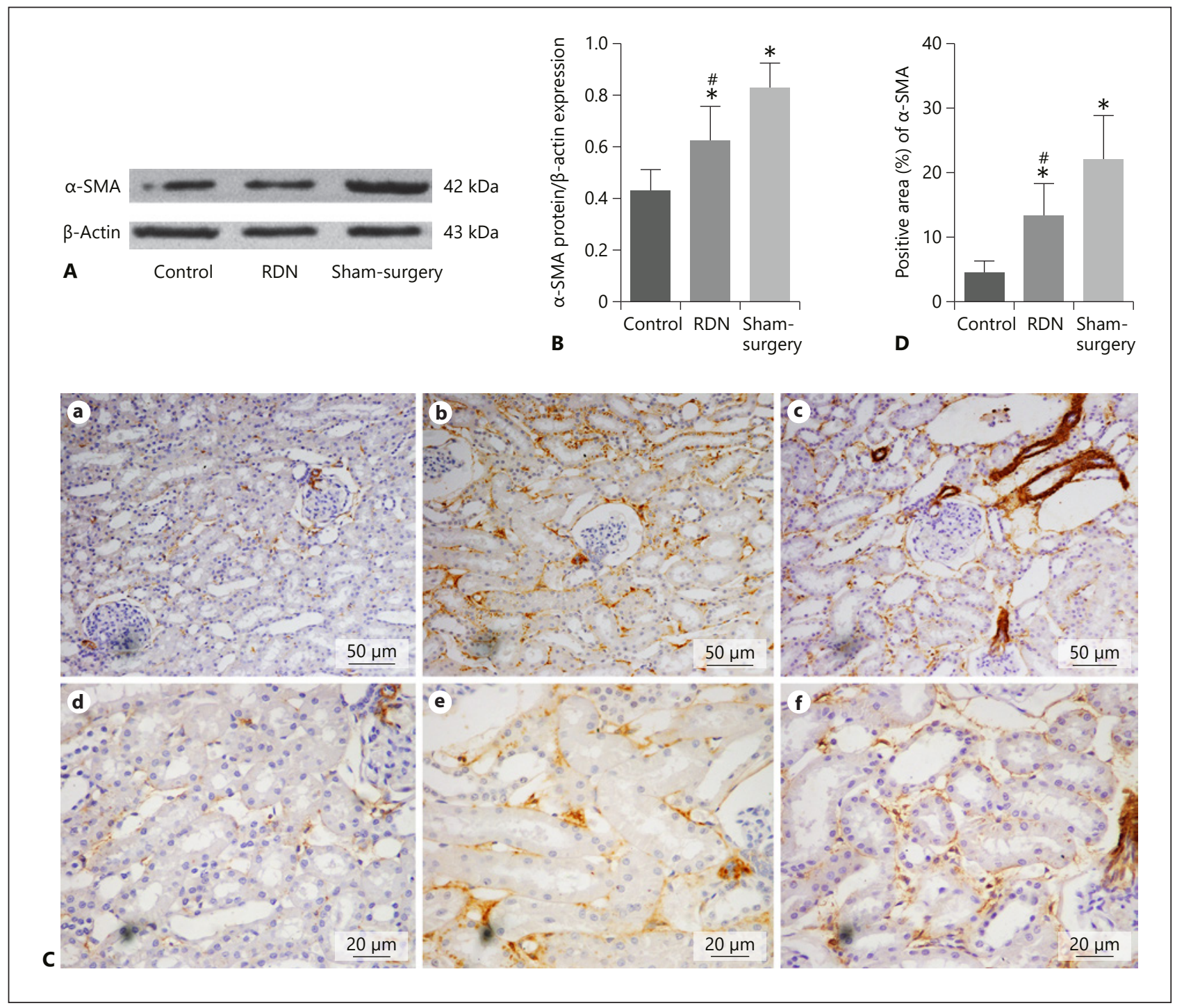

Fig. 2. Effect of RDN and high-fat diet induced hypertension on the expression of renal $\alpha$-SMA. A, B $\alpha$-SMA protein expression in canine kidney tissue in different groups is assessed by western blotting. $\mathbf{C}$ Immunohistochemistry (a-c 200x; d-f 400×) and (D) quantification results of $\alpha$-SMA in canine kidney tissue in controls, RDN, and sham-surgery groups 6 months post-surgery. $n=5$, data are presented as mean \pm SD. $* p<0.05$, vs. control group. ${ }^{\#} p<0.05$ vs. sham-surgery group. $\alpha$-SMA, alpha-smooth muscle actin; RDN, renal denervation.

Immunohistochemical results (Fig. 2C) indicated that the positive $\alpha$-SMA expression was mainly localized in the cytoplasm and membranes of renal tubular epithelial cells. Compared with the control group, both the RDN and the sham-surgery groups exhibited positive expressions, and the sham-surgery group had the strongest expression, and the positive surface density was higher than in the RDN group (Fig. 2D).

\section{Expression of E-Cadherin Protein}

As shown in Figure 3A, B and online supplementary Table 1, after RDN, there were differences in the expression of E-cadherin protein between the control group, RDN group, and the sham-surgery group. The control group showed a higher expression of E-cadherin than that in the RDN and the sham-surgery groups. 
Kidney

Blood Pressure

Research
Kidney Blood Press Res 2019;44:628-642

\begin{tabular}{l|ll}
\hline DOI: $10.1159 / 000500918$ & (c) 2019 The Author(s). Published by S. Karger AG, Basel
\end{tabular}

www.karger.com/kbr

Gong et al.: Cather-Based RDN Attenuates Kidney Interstitial Fibrosis

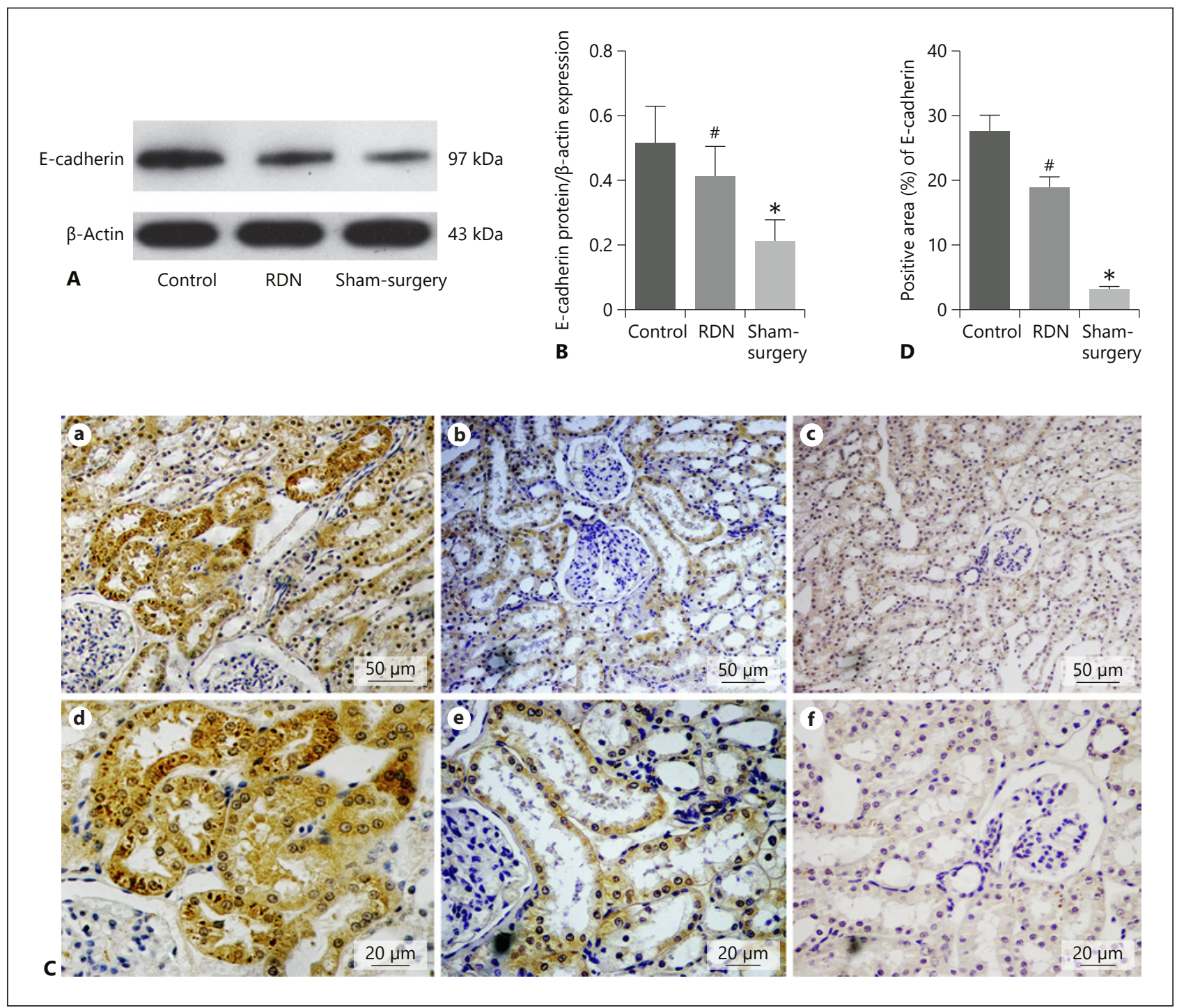

Fig. 3. Effect of RDN and high-fat diet induced hypertension on the expression of renal E-cadherin. A, B Ecadherin protein expression in canine kidney tissue in different groups is assessed by western blotting. C Immunohistochemistry (a-c 200x; d-f 400x) and (D) quantification results of E-cadherin in canine kidney tissue in Controls, RDN, and sham-surgery groups 6 months post-surgery. $n=5$, data are presented as mean \pm SD. ${ }^{*} p<0.05$, vs. control group. ${ }^{\#} p<0.05$ vs. sham-surgery group. RDN, renal denervation.

Immunohistochemical results (Fig. 3C) showed that E-cadherin immunoreactive products were mainly localized in the renal tubular epithelial cell membrane. Compared to the sham-surgery group, both the RDN group and the control group exhibited a positive expression. The control group exhibited the strongest expression. The positive surface density of the control group was higher than that of the RDN group and the sham-surgery group, and the RDN group showed a higher expression of E-cadherin than that in the shamsurgery group (Fig. 3D).

\section{Expression of CTGF Protein}

Western blot test results showed that at 6 months post-surgery, there were differences in the expression of CTGF protein among the 3 groups (Fig. 4A, B; online suppl. Table 1). The 
RDN and the sham-surgery groups showed a higher expression of CTGF than that in the control group, and the sham-surgery group exhibited the highest expression.

\section{Histological and Electron Microscopic Analysis}

Hematoxylin and eosin staining of renal tissue (Fig. 5A) showed that the renal tubules were swollen; it also showed interstitial edema and cell infiltration in sham-surgery group and RDN group. However, renal tubules were fused and necrotic in the sham-surgery group. The renal tubules in the control group showed no abnormalities. Masson staining (Fig. 5B) showed obvious collagen deposits in the renal interstitium of the sham-surgery group and RDN group. However, interstitial collagen content was most increased in the sham-surgery group (Fig. 5C).

Electron microscopic examination (Fig. 6) showed that the striated margins of renal tubular epithelial cells in the control group (Fig. 6A) were clear; in addition, edema and fibrous tissue hyperplasia were not found in the interstitium. In the RDN group, the striated margins of renal tubular epithelial cells disappeared, and some mitochondria vacuolated with focal cell infiltration (Fig. 6B). In the sham-surgery group, the epithelial cells of the renal tubules were congested and swollen, the striated margins of the cells disappeared, some epithelial cells atrophied and became necrotic, the mitochondria swelled and vacuolated in the cytoplasm, and the nuclear chromatin exhibited granules of various sizes (Fig. 6C).

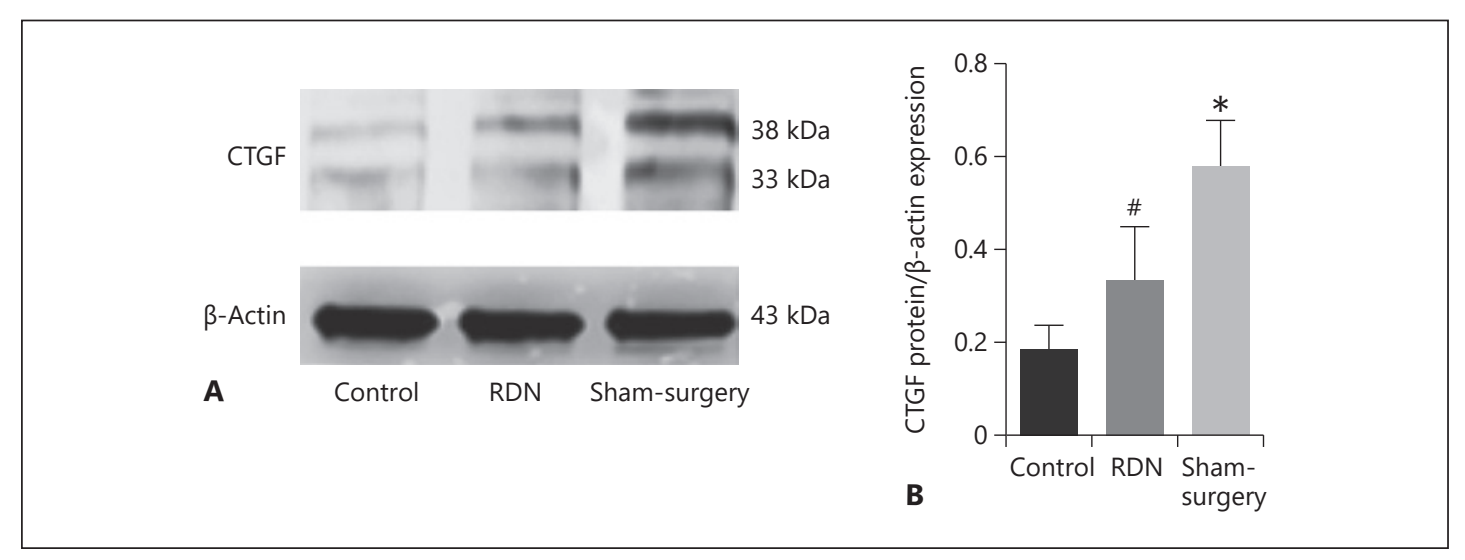

Fig. 4. Effect of RDN and high-fat diet induced hypertension on the expression of renal CTGF. A, B CTGF protein expression in canine kidney tissue in different groups is assessed by western blotting. $n=5$, data are presented as mean \pm SD. ${ }^{*} p<0.05$, vs. control group. ${ }^{*} p<0.05$ vs. sham-surgery group. CTGF, connective tissue growth factor; RDN, renal denervation.

Fig. 5. Changes in renal tubules and interstitial pathology after RDN in each group. A Hematoxylin and eosin staining of kidney tissue (a-c 200x; d-f 400x) in each group 6 months post-surgery showing increased fibrosis in the sham-surgery group. a, $\mathbf{d}$ The control group shows almost normal tubules. $\mathbf{b}$, e The RDN group shows that the renal tubular morphology is beginning to change with vacuolar degeneration in some tubular epithelial cells. c, $\mathbf{f}$ The sham-surgery group shows that visible focal vacuolization of renal tubular epithelia cells. B Masson trichrome staining of kidney tissue (a-c 200x; d-f 400x) and (C) quantification results in each group 6 months post-surgery showing increased fibrosis in the sham-surgery group. a, $\mathbf{d}$ The control group shows that renal tubular and interstitial cells without obvious collagen deposition. $\mathbf{b}$, e The RDN group shows renal tubular and interstitial cells with a small amount of collagen fiber deposition. $\mathbf{c}, \mathbf{f}$ The shamsurgery group shows that tubular and interstitial cells with obvious collagen fiber deposition. $n=8-10$, data are presented as mean \pm SD. ${ }^{*} p<0.05$, vs. control group. ${ }^{*} p<0.05$ vs. sham-surgery group.

(For figure see next page.) 
Kidney
Blood Pressure

Research
Kidney Blood Press Res 2019;44:628-642

DOI: 10.1159/000500918

(c) 2019 The Author(s). Published by S. Karger AG, Basel www.karger.com/kbr

Gong et al.: Cather-Based RDN Attenuates Kidney Interstitial Fibrosis

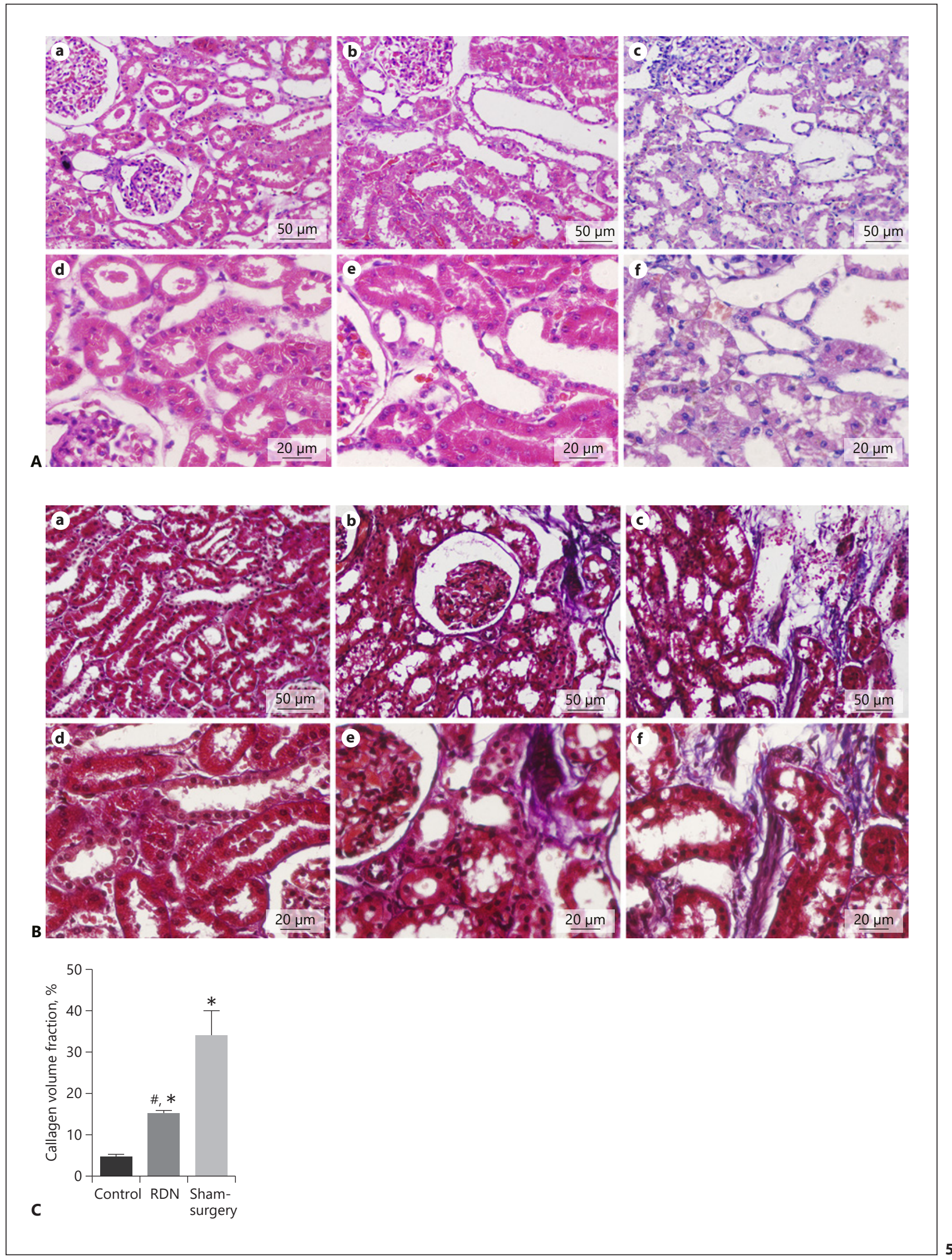




\section{Kidney \\ Blood Pressure \\ Research}

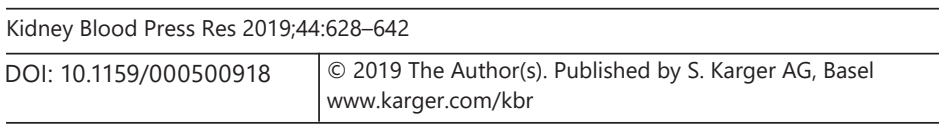

Gong et al.: Cather-Based RDN Attenuates Kidney Interstitial Fibrosis
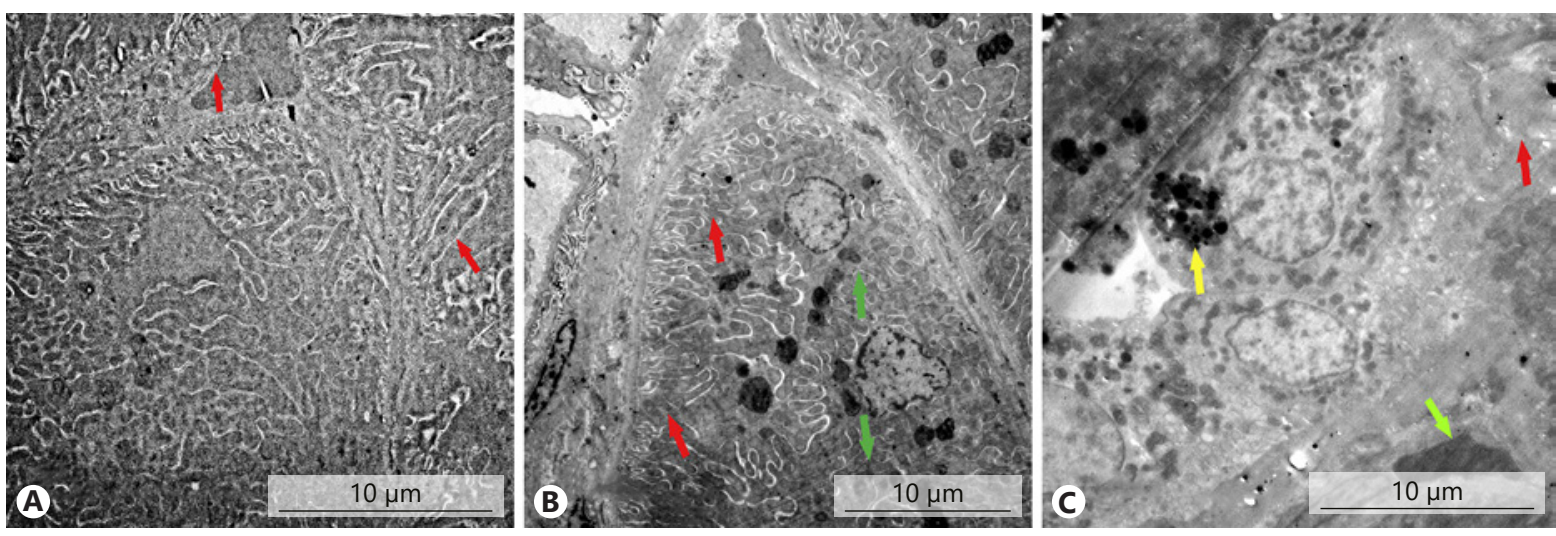

Fig. 6. Electron microscopy of renal tissue $(2,000 \times)$ in each group 6 months post-surgery. A The control group had clear striated border in renal tubular epithelial cells (red arrows), no edema, and no proliferation of fibrous tissue in the interstitium. B The RDN group had no striated border in the tubular epithelial cells (red arrows), vacuolation of intracytoplasmic mitochondria accompanied by focal cell infiltration (green arrows). C The sham-surgery group had no striated border in the tubular epithelial cells (red arrow), congestion and swelling of renal tubular epithelial cells, atrophy and necrosis in some epithelial cell, swelling and vacuolization of mitochondria in the cytoplasm (green arrow), massive irregular particle in chromatin, interstitial edema, and fibroplasia (yellow arrow).

\section{Discussion}

The present findings are in line with those of previous clinical studies that indicate the efficacy for RDN in resistant hypertension $[19,20]$. Our study showed that (1) RDN causes sustained reductions in BP in hypertension canines and (2) RDN can halt the progression of renal impairment induced by obesity and hypertension. These findings suggest that RDN can not only decrease the BP but also slowdown the progression of renal impairment and renal structural changes.

Both obesity and hypertension can cause renal disease through mechanisms such as inflammation and over activation of SNS and RAAS [3, 4, 21]. Previous studies $[1,22]$ found that obese subjects have increased SNS activity as assessed by plasma NE and tissue NE spillover; these results were consistent with ours. RAAS is a well-known regulator of BP that contributes to the onset and progression of chronic renal damage [23, 24]. RAAS activity mutually influences the secretion of neurohumoral factors, such as AngII and renin. In our study, we found that RDN suppressed the levels of circulating AngII and inhibited renal remodeling in a canine model of high-fat diet-induced hypertension. These effects might be associated with decreased activity of the SNS and RAAS after RDN.

AngII is the main effector of RAAS [25], which can directly contribute to fibrosis [23]. When AngII is activated, renal tubular epithelial cells can secrete TGF- $\beta 1$, which is a strong fibrogenic factor $[26,27]$. CTGF is an another important profibrotic mediator that acts as a downstream mediator of TGF- $\beta$-induced and AngII-induced production of the extracellular matrix (ECM) production. Moreover, CTGF can increase TGF- $\beta 1$ responses and both factors synergize to promote persistent fibrosis [8,9]. In vivo, blockade of CTGF synthesis or activity can reduce TGF- $\beta$-induced collagen synthesis. It has been shown [28] that the use of angiotension-converting enzyme inhibitors in experimental nephropathy models can reduce the production of TGF- $\beta 1$ and delay the progression of renal interstitial fibrosis. TGF- $\beta 1$-induced transdifferentiation of renal tubular epithelial cells leads to decreased expression of E-cadherin and increased expression of epidermal growth factor, resulting in the production of $\alpha$-SMA [10]. Activation of $\alpha$-SMA-positive myofibroblast cells is believed to be a central 


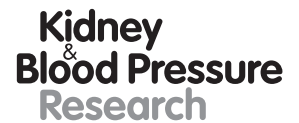

Kidney
Blood Pressure

Research \begin{tabular}{l|l}
\hline Kidney Blood Press Res 2019;44:628-642 \\
\hline DOI: 10.1159/000500918 & $\begin{array}{l}\text { O 2019 The Author(s). Published by S. Karger AG, Basel } \\
\text { www.karger.com/kbr }\end{array}$ \\
\hline
\end{tabular}

Gong et al.: Cather-Based RDN Attenuates Kidney Interstitial Fibrosis

event that has a key role in the progression of chronic renal fibrosis. E-cadherin is an important adhesion molecule in the renal tubular epithelial cells. It is the ligament that constitutes the tight junction between renal tubular epithelial cells. When E-cadherin expression was inhibited, the tight junction between the cells disappeared, the basement membrane was disrupted, and the renal tubular epithelial cells transformed into myofibroblasts.

Renal interstitial fibrosis is a common pathological feature of renal disease progression and end-stage renal insufficiency due to various causes [28]. Its outcomes include progressive and irreversible impairment of renal function. Renal interstitial fibrosis damages renal tubular epithelial cells, activates fibrogenic factors, increases ECM synthesis, and decreases its degradation, resulting in the excessive deposits of the ECM in the renal interstitium and proliferation of renal interstitial fibroblasts [29]. Studies [30, 31] have shown that renal tubular epithelial cell transdifferentiation has an important role in renal interstitial fibrosis. Epithelial cell transdifferentiation involves decreased or the disappearance of epithelial cell adhesion, expression of $\alpha$-SMA, destruction of the renal tubular basement membrane, and cell migration. Renal tubular epithelial cells can be transdifferentiated into myofibroblasts via epithelial cell transdifferentiation.

Myofibroblasts enter the mesenchyme through the destroyed basement membrane and synthesize ECM in the renal interstitium, thus leading to renal interstitial fibrosis. Myofibroblasts also have the characteristics of fibroblasts and myocytes and express $\alpha$-SMA. Ng et al. [32] found some renal tubular epithelial cells expressing $\alpha$-SMA in renal fibrosis models established by $5 / 6$ nephrectomy. These results confirmed that activated RAAS can promote the production of TGF- $\beta 1$, induce the transdifferentiation of renal tubular epithelial cells, and ultimately promote the formation of renal interstitial fibrosis. The results of previous studies and ours suggest that blocking renal sympathetic activity could be a novel therapeutic strategy for treating high-fat diet-induced hypertension.

The renal tubular function test showed that urinary albumin in the sham-surgery group continued to increase after RDN. This increase in the RDN group was slower than that in the sham-surgery group, and there was no significant difference in the increase between the RDN group before or after RDN, suggesting that although RDN can delay the progression of tubular dysfunction, it cannot reverse the impaired tubular function.

There were no significant difference between male and female beagles on BP, the HR, NE, AngII, urine protein, and the expression of TGF- $\beta 1, \alpha$-SMA, E-cadherin and CTGF protein in our study with regard to the effect of RDN, which was in line with the previous findings [3335]. Our study suggests that the renal nerves contribute equally to the high-fat diet-induced hypertension in male and female beagles and is independent of the influence of gender on BP and kidney interstitial fibrosis.

In our study, RDN reduced arterial pressure, reduced the deterioration of renal function, and prevented an ameliorated kidney interstitial fibrosis in a canine model of high-fat dietinduced hypertension. RDN may be a novel therapeutic approach to obesity and CKD. BP control is mandatory for slowing the progression of kidney disease. However, currently available therapeutic efforts are insufficient to halt progression of renal impairment, and alternative treatment options are warranted. Therefore, inhibition of sympathetic nerve activity may be such a potential therapeutic option.

\section{Limitations}

Sufficient attention was not given to the details of this experiment, which resulted in the accidental death of experimental animals and a smaller sample size. Because of the small sample size, the animals were not sacrificed at 1 and 3 months after RDN so that changes in the renal tissue could be observed and compared with those at 6 months after RDN. There were no data regarding 24-h urine protein before and after surgery; therefore, changes in the 
urinary albumin levels could not be evaluated. We only had vesicopuncture urine samples available to test the urinary albumin levels before and after surgery. In this study, we only reported the indices in vivo and did not further verify whether the local NE level could affect the expressions of TGF- $\beta 1, \alpha$-SMA, CTGF, and E-cadherin by the RAAS pathway in vitro.

\section{Conclusions}

Briefly, RDN had an obvious effect against hypertension. Blocking the renal sympathetic nerves can prevent the activation of RAAS, which is mediated by these sympathetic nerves, and further prevent the progression of renal fibrosis induced by RAAS, thus providing an experimental and theoretical basis for delaying the progress of slow renal insufficiency after RDN. The mechanism of RDN is multifactorial, and the duration of hypotension, outcomes, and maintenance of kidney function are prolonged. However, the period of impact requires more attention and needs more in-depth and long-term research.

\section{Acknowledgments}

This study was supported by the Independent Exploration and Innovation Project for Postgraduates of Central South University (grant number 2016zzts154).

\section{Statement of Ethics}

This study was conducted in compliance with the guidelines of the National Institutes of Health for the care and use of laboratory animals and approved by the animal studies subcommittee of the Third Xiangya Hospital of Central South University.

\section{Disclosure Statement}

The authors of this manuscript state that they do not have any conflict of interests and nothing to disclose.

\section{Author Contributions}

L.G., F.J., and Z.Z.: designed the research. L.G., Z.Z., X.W., H.L., and Y.K.: performed the research. L.G. and F.J.: analyzed the data. L.G. and G.Y.: wrote the manuscript.

\section{References}

1 Hall JE, Crook ED, Jones DW, Wofford MR, Dubbert PM. Mechanisms of obesity-associated cardiovascular and renal disease. Am J Med Sci. 2002 Sep;324(3):127-37.

2 Hall JE, Henegar JR, Dwyer TM, Liu J, Da Silva AA, Kuo JJ, et al. Is obesity a major cause of chronic kidney disease? Adv Ren Replace Ther. 2004 Jan;11(1):41-54.

3 Hall ME, do Carmo JM, da Silva AA, Juncos LA, Wang Z, Hall JE. Obesity, hypertension, and chronic kidney disease. Int J Nephrol Renovasc Dis. 2014 Feb;7:75-88.

4 Henegar JR, Bigler SA, Henegar LK, Tyagi SC, Hall JE. Functional and structural changes in the kidney in the early stages of obesity. J Am Soc Nephrol. 2001 Jun;12(6):1211-7.

5 Nath KA. The tubulointerstitium in progressive renal disease. Kidney Int. 1998 Sep;54(3):992-4.

6 Ruiz-Ortega M, Rupérez M, Esteban V, Rodríguez-Vita J, Sánchez-López E, Carvajal G, et al. Angiotensin II: a key factor in the inflammatory and fibrotic response in kidney diseases. Nephrol Dial Transplant. 2006 Jan;21(1): $16-20$. 


\begin{tabular}{l|l}
\hline Kidney Blood Press Res 2019;44:628-642 \\
\hline DOI: 10.1159/000500918 & $\begin{array}{l}\text { (c) 2019 The Author(s). Published by S. Karger AG, Basel } \\
\text { www.karger.com/kbr }\end{array}$ \\
\hline
\end{tabular}

Gong et al:: Cather-Based RDN Attenuates Kidney Interstitial Fibrosis

7 Bhowmick NA, Ghiassi M, Bakin A, Aakre M, Lundquist CA, Engel ME, et al. Transforming growth factor-beta1 mediates epithelial to mesenchymal transdifferentiation through a RhoA-dependent mechanism. Mol Biol Cell. 2001 Jan;12(1):27-36.

8 Rupérez M, Lorenzo O, Blanco-Colio LM, Esteban V, Egido J, Ruiz-Ortega M. Connective tissue growth factor is a mediator of angiotensin II-induced fibrosis. Circulation. 2003 Sep;108(12):1499-505.

9 Ruiz-Ortega M, Rodríguez-Vita J, Sanchez-Lopez E, Carvajal G, Egido J. TGF-beta signaling in vascular fibrosis. Cardiovasc Res. 2007 May;74(2):196-206.

10 Yang J, Liu Y. Blockage of tubular epithelial to myofibroblast transition by hepatocyte growth factor prevents renal interstitial fibrosis. J Am Soc Nephrol. 2002 Jan;13(1):96-107.

11 Davis MI, Filion KB, Zhang D, Eisenberg MJ, Afilalo J, Schiffrin EL, et al. Effectiveness of renal denervation therapy for resistant hypertension: a systematic review and meta-analysis. J Am Coll Cardiol. 2013 Jul;62(3): 231-41.

12 Steigerwald K, Titova A, Malle C, Kennerknecht E, Jilek C, Hausleiter J, et al. Morphological assessment of renal arteries after radiofrequency catheter-based sympathetic denervation in a porcine model. J Hypertens. 2012 Nov;30(11):2230-9.

13 Schlaich MP, Socratous F, Hennebry S, Eikelis N, Lambert EA, Straznicky N, et al. Sympathetic activation in chronic renal failure. J Am Soc Nephrol. 2009 May;20(5):933-9.

14 Veelken R, Vogel EM, Hilgers K, Amann K, Hartner A, Sass G, et al. Autonomic renal denervation ameliorates experimental glomerulonephritis. J Am Soc Nephrol. 2008 Jul;19(7):1371-8.

15 Amann K, Rump LC, Simonaviciene A, Oberhauser V, Wessels S, Orth SR, et al. Effects of low dose sympathetic inhibition on glomerulosclerosis and albuminuria in subtotally nephrectomized rats. J Am Soc Nephrol. 2000 Aug;11(8):1469-78.

16 Veelken R, Schmieder RE. Renal denervation-implications for chronic kidney disease. Nat Rev Nephrol. 2014 Jun;10(6):305-13.

17 Zhang Z, Yang K, Zeng L, Wang X, Jiang F, Tu S, et al. Renal simplicity denervation reduces blood pressure and renal injuries in an obesity-induced hypertension dog model. Clin Exp Pharmacol Physiol. 2017 Dec;44(12): 1213-23.

18 Chen W, Tang X, Yang X, Weng C, Yang K, Wen J, et al. Effects and Mechanisms of Radiofrequency Ablation of Renal Sympathetic Nerve on Anti-Hypertension in Canine. Arq Bras Cardiol. 2017 Mar;108(3):237-45.

19 Krum H, Schlaich MP, Sobotka PA, Böhm M, Mahfoud F, Rocha-Singh K, et al. Percutaneous renal denervation in patients with treatment-resistant hypertension: final 3-year report of the Symplicity HTN-1 study. Lancet. 2014 Feb;383(9917):622-9.

20 Esler MD, Böhm M, Sievert H, Rump CL, Schmieder RE, Krum H, et al. Catheter-based renal denervation for treatment of patients with treatment-resistant hypertension: 36 month results from the SYMPLICITY HTN-2 randomized clinical trial. Eur Heart J. 2014 Jul;35(26):1752-9.

21 Underwood PC, Adler GK. The renin angiotensin aldosterone system and insulin resistance in humans. Curr Hypertens Rep. 2013 Feb;15(1):59-70.

22 Hall JE, da Silva AA, do Carmo JM, Dubinion J, Hamza S, Munusamy S, et al. Obesity-induced hypertension: role of sympathetic nervous system, leptin, and melanocortins. J Biol Chem. 2010 Jun;285(23):17271-6.

23 Kelly TN, Raj D, Rahman M, Kretzler M, Kallem RR, Ricardo AC, et al.; CRIC Study Investigators. The role of renin-angiotensin-aldosterone system genes in the progression of chronic kidney disease: findings from the Chronic Renal Insufficiency Cohort (CRIC) study. Nephrol Dial Transplant. 2015 Oct;30(10):1711-8.

24 Remuzzi G, Perico N, Macia M, Ruggenenti P. The role of renin-angiotensin-aldosterone system in the progression of chronic kidney disease. Kidney Int Suppl. 2005 Dec;68(99):S57-65.

25 Aros C, Remuzzi G. The renin-angiotensin system in progression, remission and regression of chronic nephropathies. J Hypertens Suppl. 2002 Jun;20(3):S45-53.

26 Kanwar YS. TGF- $\beta$ and renal fibrosis: a Pandora's box of surprises. Am J Pathol. 2012 Oct;181(4):1147-50.

27 Loeffler I, Wolf G. Transforming growth factor- $\beta$ and the progression of renal disease. Nephrol Dial Transplant. 2014 Feb;29(Suppl 1):i37-45.

28 Eddy AA. Molecular basis of renal fibrosis. Pediatr Nephrol. 2000 Dec;15(3-4):290-301.

29 Razzaque MS, Taguchi T. Cellular and molecular events leading to renal tubulointerstitial fibrosis. Med Electron Microsc. 2002 Jun;35(2):68-80.

30 Hou FF, Liu Y. New insights into the pathogenesis and therapeutics of kidney fibrosis. Kidney Int Suppl (2011). 2014 Nov;4(1):1-1.

31 Lan HY. Tubular epithelial-myofibroblast transdifferentiation mechanisms in proximal tubule cells. Curr Opin Nephrol Hypertens. 2003 Jan;12(1):25-9.

32 Ng YY, Huang TP, Yang WC, Chen ZP, Yang AH, Mu W, et al. Tubular epithelial-myofibroblast transdifferentiation in progressive tubulointerstitial fibrosis in 5/6 nephrectomized rats. Kidney Int. 1998 Sep;54(3):86476.

33 Iliescu R, Yanes LL, Bell W, Dwyer T, Baltatu OC, Reckelhoff JF. Role of the renal nerves in blood pressure in male and female SHR. Am J Physiol Regul Integr Comp Physiol. 2006 Feb;290(2):R341-4.

34 Prochnau D, Heymel S, Göbel B, Figulla HR, Surber R. Resistant hypertension: multivariate predictors of blood pressure response to renal denervation. Int J Cardiol. 2013 Oct;168(3):3130-2.

35 Choi SY, Hwang JS, Kim IH, Hwang DY, Kang HG. Basic data on the hematology, serum biochemistry, urology, and organ weights of beagle dogs. Lab Anim Res. 2011 Dec;27(4):283-91. 